\title{
Inflammatory arthritis in children with osteochondrodysplasias
}

\begin{abstract}
Rosie Scuccimarri, E Michel Azouz, Karen N Watanabe Duffy, François Fassier, Ciarán M Duffy
\end{abstract}

\begin{abstract}
Division of
Rheumatology, Montreal Children's

Hospital, Montreal,

Quebec, Canada

R Scuccimarri

K N Watanabe Duffy

C M Duffy
\end{abstract}

Division of Radiology,

Montreal Children's

Hospital

E M Azouz

Division of

Orthopaedic Surgery,

Montreal Children's

Hospital

F Fassier

Department of

Paediatrics, McGill

University, Montreal,

Quebec, Canada

R Scuccimarri

K N Watanabe Duffy

C M Duffy

Department of

Radiology, McGill

University

E M Azouz

Department of

Surgery, McGill

University

F Fassier

Correspondence to:

Dr CM Duffy, Division of

Rheumatology, Montreal

Children's Hospital, 2300

Tupper street, Room C503,

Montreal, Quebec, Canada

$\mathrm{H} 3 \mathrm{H} 1 \mathrm{P} 3$

Email:

ciaran.duffy@muhc.mcgill.ca

Accepted for publication

10 April 2000

\begin{abstract}
Osteochondrodysplasias are a heterogeneous group of genetic skeletal dysplasias. Patients with these diseases commonly develop an early degenerative arthritis or osteoarthritis. Occasional observations of inflammatory arthritis have been made in this population but such observations are based on clinical grounds alone without confirmatory imaging studies. Four patients followed up in a paediatric rheumatology clinic with three different skeletal dysplasias, who had both clinical and radiological evidence of an inflammatory arthritis and coexistent degenerative arthritis, are described.

(Ann Rheum Dis 2000;59:864-869)
\end{abstract}

Osteochondrodysplasias are a heterogeneous group of genetic skeletal dysplasias. ${ }^{12}$ These diseases are diagnosed and classified by their clinical picture, radiographic findings, and their genetic inheritance. ${ }^{1}$ Patients with these diseases commonly develop an early degenerative arthritis or osteoarthritis, which is believed to occur because of abnormal mechanical factors or abnormalities in the articular cartilage, or both. ${ }^{13}$ There have also been reports of inflammatory arthritis in this group, characterised by joint effusions and soft tissue swelling. ${ }^{2-8}$ These observations have been merely clinical. Some authors have suggested that the disease in these patients may resemble rheumatoid arthritis clinically but that they do not have the typical radiographic changes. ${ }^{78}$ Patients described in the literature have not been shown to have any radiographic signs of bone destruction, ${ }^{5-10}$ and the degree of soft tissue swelling on plain radiographs has not been as marked as in those with a true inflammatory disease. ${ }^{8}$ Additionally, laboratory findings are not consistent with systemic inflammation. ${ }^{246-8}$ Synovial biopsies have also been normal. ${ }^{7} 910$

We describe here four patients with three different skeletal dysplasias, followed up in our paediatric rheumatology clinic, who have had both clinical and radiological evidence of inflammatory arthritis in addition to clinical and radiological evidence of degenerative arthritis.

\section{Case reports}

Table 1 summarises the data on these four cases.

\section{CASE 1}

Patients 1 and 2 are siblings who have been previously reported on by Lewkonia and BechHansen. ${ }^{2}$ They were born in the Azores, Portugal. Their parents are not consanguineous.

Patient No 1 is now aged 16 years. She was first noted to have pain and stiffness in her interphalangeal finger joints at the age of 3 months after a febrile illness. By 8 months of age she had further joint disease and juvenile rheumatoid arthritis (JRA) was diagnosed. At age 7 years the family moved to Canada where spondyloepiphysial dysplasia tarda (also referred to as progressive pseudorheumatoid arthropathy) was diagnosed. This was based on her radiological findings and the pattern of inheritance, which was autosomal recessive. She was treated with a non-steroidal antiinflammatory drug (NSAID) and physiotherapy. She transferred to our institution at age 13, where she continues to be followed up.

Our initial examination showed a nondysmorphic adolescent. Her height was on the

Table 1 Summary of evidence supporting an inflammatory arthritis

\begin{tabular}{|c|c|c|c|c|}
\hline Cases & History & Clinical examination & Plain radiographs & $M R I^{\star}$ of hips \\
\hline 1 & $\begin{array}{l}\text { - Morning stiffness } \\
\text { - Stiffness after inactivity } \\
\text {-Joint swelling }\end{array}$ & $\begin{array}{l}\bullet \text { Polyarthritis with effusive disease (small and large } \\
\text { joints) }\end{array}$ & $\begin{array}{l}\text { - Soft tissue swelling } \\
\text { - Diffuse osteopenia } \\
\text { - Joint space narrowing } \\
\text { - Bony overgrowth } \\
\text { - Hip erosions }\end{array}$ & $\begin{array}{l}\text { Effusions } \\
\bullet \text { Erosions } \\
\bullet \text { After gadolinium: synovitis }\end{array}$ \\
\hline 2 & $\begin{array}{l}\text { - Morning stiffness } \\
\text { - Stiffness after inactivity } \\
\text {-Joint swelling }\end{array}$ & $\begin{array}{l}\text {-Polyarthritis with effusive disease (small and large } \\
\text { joints) }\end{array}$ & $\begin{array}{l}\text { - Soft tissue swelling } \\
\text { - Diffuse osteopenia } \\
\text { - Joint space narrowing } \\
\text { - Hip and wrist } \\
\text { erosions }\end{array}$ & $\begin{array}{l}\bullet \text { Effusions } \\
\text { • Erosions } \\
\text { - After gadolinium: synovitis }\end{array}$ \\
\hline 3 & $\bullet$ Morning stiffness & $\begin{array}{l}\text {-Stress pain and decreased range of movement at } \\
\text { the hips }\end{array}$ & $\begin{array}{l}\bullet \text { Joint space narrowing } \\
\bullet \text { Osteopenia }\end{array}$ & $\begin{array}{l}\bullet \text { Effusions } \\
\text {-After gadolinium: synovitis and large } \\
\text { erosion }\end{array}$ \\
\hline 4 & $\bullet$ Morning stiffness & -Soft tissue swelling of the knee & $\begin{array}{l}\text {-Unremarkable } \\
\bullet \text { Osteopenia }\end{array}$ & $\begin{array}{l}\text { •Effusion } \\
\text { •After gadolinium: synovitis }\end{array}$ \\
\hline
\end{tabular}

${ }^{\star} \mathrm{MRI}=$ magnetic resonance imaging 
10 th centile and weight on the 25 th centile. She had micrognathia and limited range of movement (ROM) of her cervical spine. She had effusions of several small and large joints in her arms, but these joints were not warm. There were mild flexion deformities of her fingers, wrists, and elbows. Her hips were affected most severely, with flexion limited to 85 degrees bilaterally, and the presence of hip flexion contractures of 20 degrees bilaterally. She had marked limitation of abduction, internal and external rotation. There was a small effusion of her left knee with a mild flexion contracture of 10 degrees bilaterally. Her ankles were effused, but she maintained good ROM. An examination of the spine was unremarkable.

Radiographic examination was consistent with findings seen in spondyloepiphysial dysplasia. She had mild irregularity of the contours of the lumbar vertebral bodies of L1 to L3 with some anterior wedging of L3. She had bilateral coxa vara deformities with flattening of the epiphyses of the femoral heads. Her metacarpal joints were short with widening and flattening of the epiphyses. These epiphysial changes were also seen at the wrists and at the knees with flattening of the femoral condyles and tibial plateau bilaterally.

On plain radiographs, she was noted to have diffuse osteopenia, decreased height of the carpus, soft tissue swelling of both wrists, and hypertrophy of the ulnar styloid processes. There was narrowing of the patellofemoral joints, synovial thickening or effusions of both knees and elbows, and prominence of the capitulum of the elbows. The plain radiographs of the hips showed loss of joint space, erosions of the acetabulum and femoral head and neck, whiskering at the femoral head margins, and an osteophyte at the right acetabular roof (fig 1). Small vertebral bodies with loss of the normal lordosis at the cervical spine and normal sacroiliac joints were also seen. Computed tomographic (CT) scan of her hips showed severe, bilateral erosive arthritis of both hips including the femoral head and neck, and acetabulum. Magnetic resonance imaging (MRI) with gadolinium showed evidence of hip synovitis with thick irregular and enhancing synovium, and bilateral hip joint effusions (fig 2). Articular cartilage of the femoral head and acetabulum were irregular with erosions, and the femoral head and neck were deformed.

Her erythrocyte sedimentation rate (ESR) was unremarkable and ranged between 12 and $17 \mathrm{~mm} / 1 \mathrm{st} \mathrm{h}$. She was antinuclear antibody (ANA) and rheumatoid factor (RF) negative. She had genetic studies which showed no structural rearrangement or other alterations of the type II procollagen gene (COL2A1). ${ }^{2}$ Over the years this patient has had mostly inflammatory-type symptoms, such as morning stiffness and stiffness after inactivity, which worsen when she stops taking NSAIDs. Her pain often lessens with activity. Occasionally, she has also had mechanical-type complaints, such as an increase in pain at the end of the day and night pain. While taking NSAIDs, she was less symptomatic but had further progression and loss of ROM of her hips. Therefore, treat-

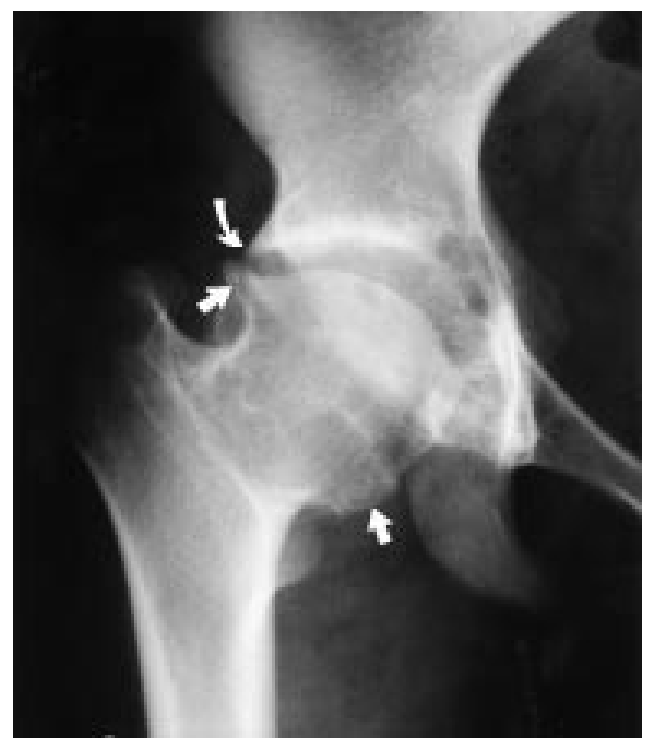

Figure 1 Patient No 1 aged 14 years. Plain film of the right hip: flattened and widened epiphysis; osteophyte (curved arrow); whiskering (straight arrows).

ment was started with low dose methotrexate. With this treatment, she had a further decrease in symptoms and for the first time gained some ROM in her wrists and hips. Her condition was stable for 10 months but, unfortunately, the disease has recently flared, and we have since increased the dose of methotrexate. At the time of the flare, an MRI with gadolinium of the hips showed bilateral thick, irregular enhancing synovium with effusions, bursitis, and bilateral femoral head and acetabular erosions more extensive than previously seen.

CASE 2

Patient No 2 (sibling of patient 1) is now 13 years old. His parents first noted a problem at the age of 2, but no diagnosis was made. $\mathrm{He}$ was seen at the age of 5 in Canada, where the diagnosis of spondyloepiphysial dysplasia tarda was made. He too was treated with an NSAID and physiotherapy. We saw him for the first time at age 11 years. On examination, he was not dysmorphic. His height was on the 10th centile, and his weight was on the 25 th centile. $\mathrm{He}$ had effusions of several small and large joints of his arms. There were 10-15 degree flexion contractures of his elbows bilaterally. His hips were also most severely affected, with marked limitation of internal and external rotation bilaterally. He had effusions in both knees and ankles but, ROM was maintained. An examination of the spine was unremarkable.

Radiographic examination was also consistent with spondyloepiphysial dysplasia, with milder changes than his sister. He had subtle irregularities of the contour of the lumbar vertebral bodies of L3, L4, and, to a lesser extent, L5. He also had minimum end plate irregularities in the lower thoracic region. He had flattening of the femoral, humeral, and metacarpal heads, as well as the femoral condyles. He had bilateral coxa vara deformities.

On plain radiographs, he was also noted to have diffuse osteopenia, decreased height of the 


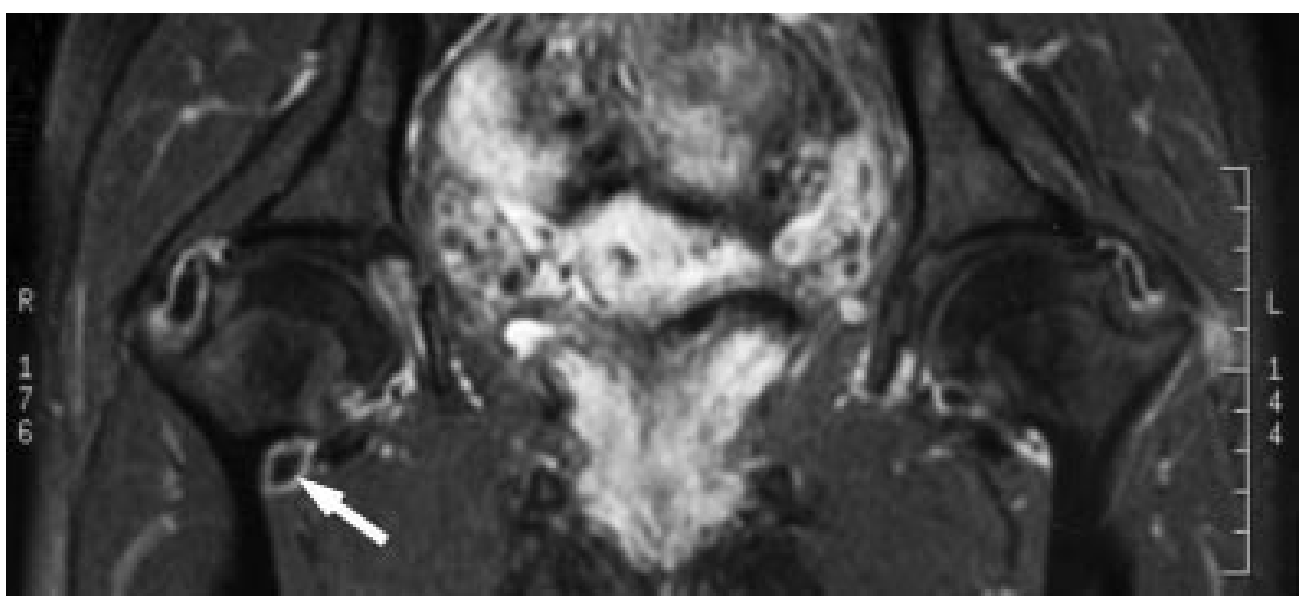

Figure 2 Patient No 1 aged 14 years. Gadolinium enhanced magnetic resonance imaging (T1 fat suppressed) of hips-coronal view: effusion (black area indicated by arrow) delineated by the enhanced and mildly thickened synovium (white areas). The changes are bilateral.

carpus, soft tissue swelling of both wrists (fig $3 \mathrm{~A})$, erosions of the left hamate and capitate (fig 3B), a large geode in the left olecranon process, synovial thickening or effusions of both knees, an osteophyte of the left acetabular roof, and bilateral, symmetrical erosions of the acetabulum. A CT scan also showed bilateral acetabular and femoral head erosions. On MRI, moderate to large hip effusions were seen bilaterally as well as irregularities of the acetabular roofs and mild femoral head flattening. Mild synovial enhancement was seen after gadolinium injection.

His ESR ranged between 16 and $22 \mathrm{~mm} / 1 \mathrm{st}$ h. He was ANA and RF negative. Genetic testing for the type II procollagen gene (COL2A1) was also negative. ${ }^{2}$

While taking NSAIDs, he has had minimum complaints. Like his sister, he has also had a mixture of inflammatory and mechanical-type symptoms, though his inflammatory symptoms predominate. He has had morning stiffness and stiffness after inactivity, especially in his fingers, but occasionally he complains of joint

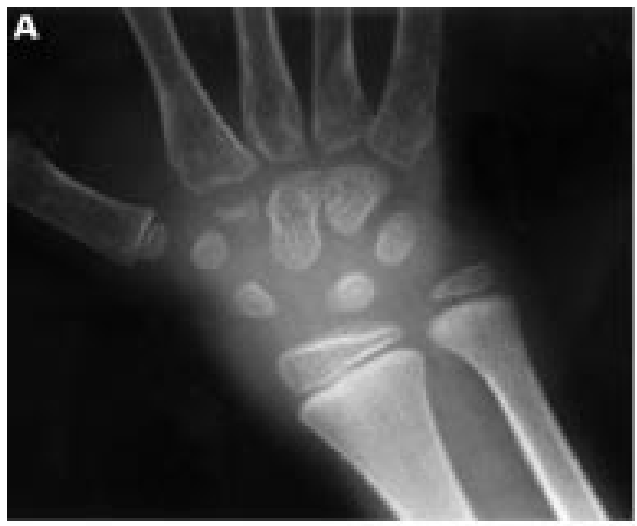

pain that worsens with activity. These symptoms are responsive to NSAIDs.

Unfortunately, while taking NSAIDs, his disease has progressed, with loss of ROM in his hips. He underwent valgus osteotomies of both hips to correct the coxa vara deformities. A histopathological examination of a femoral biopsy specimen taken at the time of surgery was unremarkable, and the growth plate at the greater trochanter showed normal cartilage. His left knee was aspirated and a synovial biopsy was also done. The fluid was noninflammatory with 217 white blood cells, $42 \%$ of which were polymorphonuclear cells. Synovial biopsy showed little chronic inflammation.

He showed some improvement postoperatively but, later, his hips deteriorated further. Therefore treatment was started with methotrexate. Since then, he has been less symptomatic and has gained some ROM at the hips.

CASE 3

Patient No 3 is an 11 year old girl with Stickler's syndrome. This is an autosomal

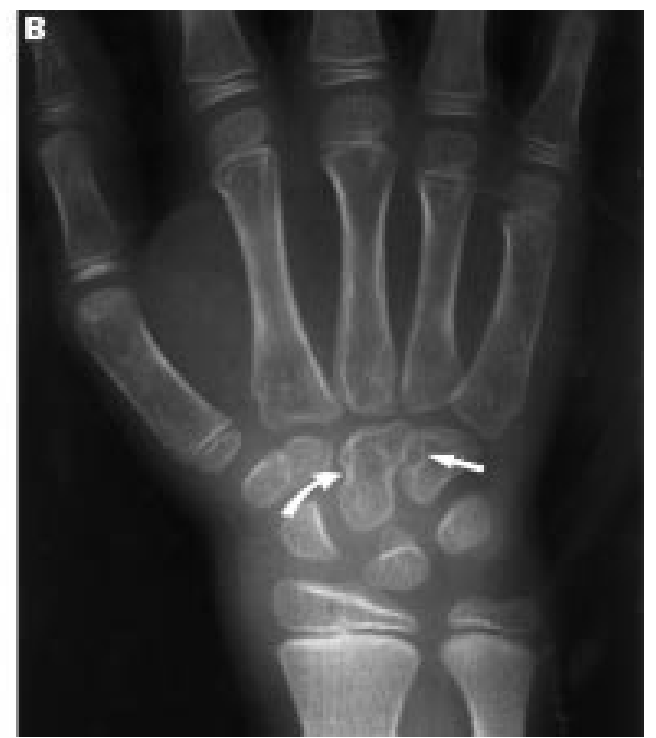

Figure 3 (A) Patient No 2 aged 8 years. Plain film of the left wrist: soft tissue swelling. (B) Patient No 2 aged 10 years. Plain film of the left hand and wrist: flattened and widened metacarpal head epiphyses; soft tissue swelling of the wrist; erosions of the hamate (straight arrow) and capitate (curved arrow); diffuse osteopenia. 

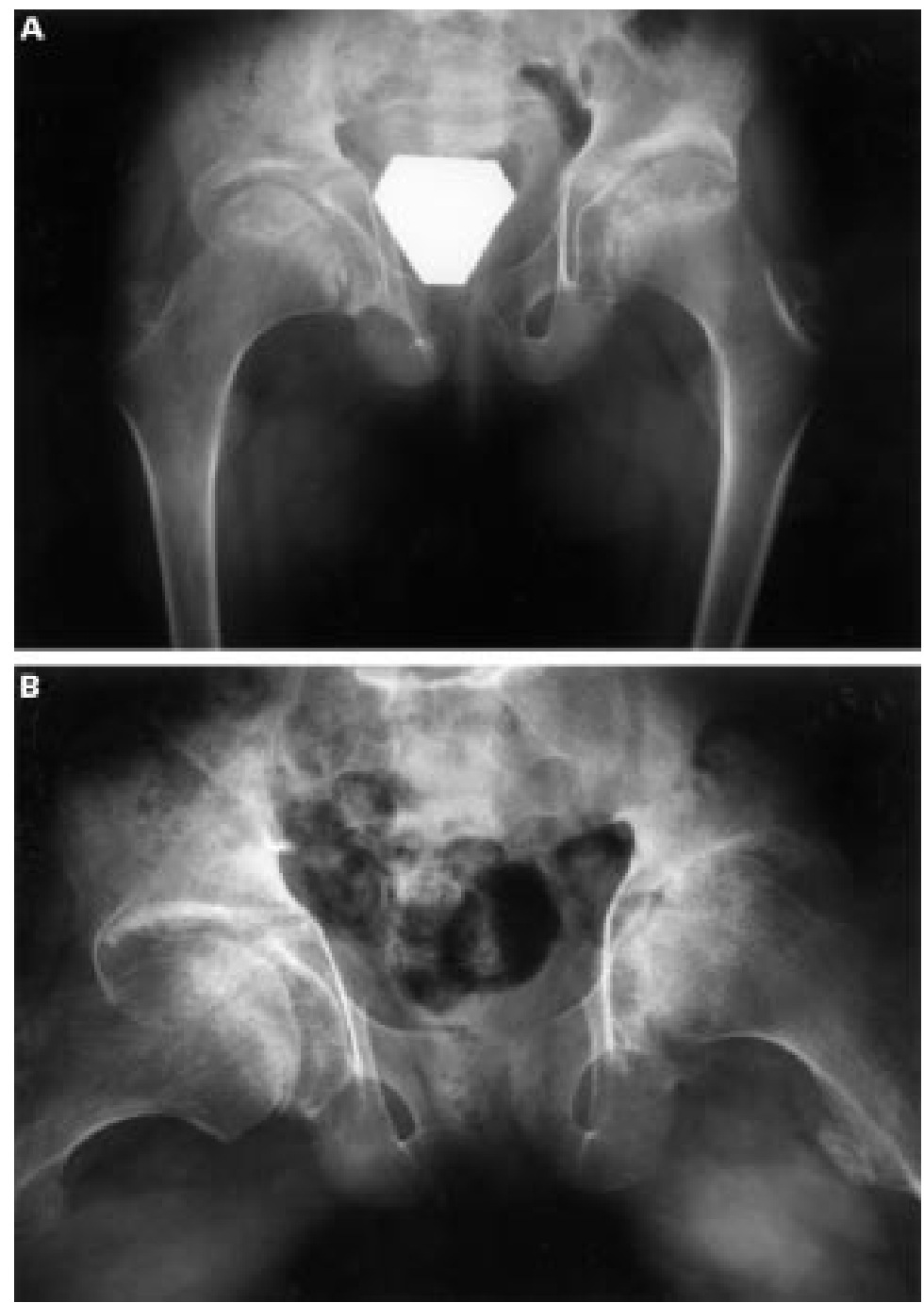

Figure 4 Patient No 3 aged 10 years. Plain films of hips: flattened and widened femoral heads; joint space narrowing and irregularity; osteopenia. (B) Patient No 3 aged 11 years. Plain films of hips: sclerosis of left hip joint; very significant joint space narrowing (rapid progression in 10 months in comparison with fig $4 A$ ); erosion of left femoral head.

dominantly inherited syndrome characterised by epiphysial dysplasia and ocular and facial abnormalities. ${ }^{11-13}$ She has myopia, cleft palate, hearing loss, and joint deformities. She was first assessed by us in August 1996 for significant morning stiffness. Her biggest problem was with her hips that led to difficulty climbing stairs and running.

On examination, her height and weight were on the 10 th centile. She was noted to have a psoriaform rash on her arm. She did not have nail pits. She had marked limitation in ROM of both hips.

Radiographic examination showed platyspondyly, widening of the pubic and ischial bones with protrusio acetabuli, large metacarpal heads, flattening of the radial heads, and bony enlargement with irregular epiphyses of the hips, ankles, and knees with secondary changes in the corresponding metaphyses.

On plain films, she was also noted to have osteopenia, irregular surfaces of the posterior facet joints of the vertebrae of $\mathrm{C} 2$ to $\mathrm{C} 7$, rapid progression of left hip joint space narrowing with irregularities and suggestion of an erosion on the left femoral head (figs $4 \mathrm{~A}$ and B). On bone scan, she had uptake in both hips, left more than right. On MRI, she had small bilateral hip joint effusions. There were irregularities of both femoral heads and loss of articular cartilage. After gadolinium, marked enhancement of the synovium of both hip joints and a large erosion over the convexity of the left femoral head was seen (fig 5).

Her ESR had been unremarkable and ranged from 13 to $19 \mathrm{~mm} / 1 \mathrm{st} \mathrm{h}$. She had a positive ANA titre of $1 / 160$, with a homogeneous pattern. She was RF negative.

Initially, she had mostly inflammatory-type symptoms that improved temporarily with naproxen. Eventually, she required intraarticular corticosteroid injection of both hips. During this procedure, it was noted that she had a large effusion of the left hip. She had some benefit from the injection but it was short lived-only five months. Treatment was later changed to indometacin which, initially, had a dramatic functional improvement. She has since worsened. Her pain has become mostly mechanical, with pain throughout the day and worse with activity. She has required a left total

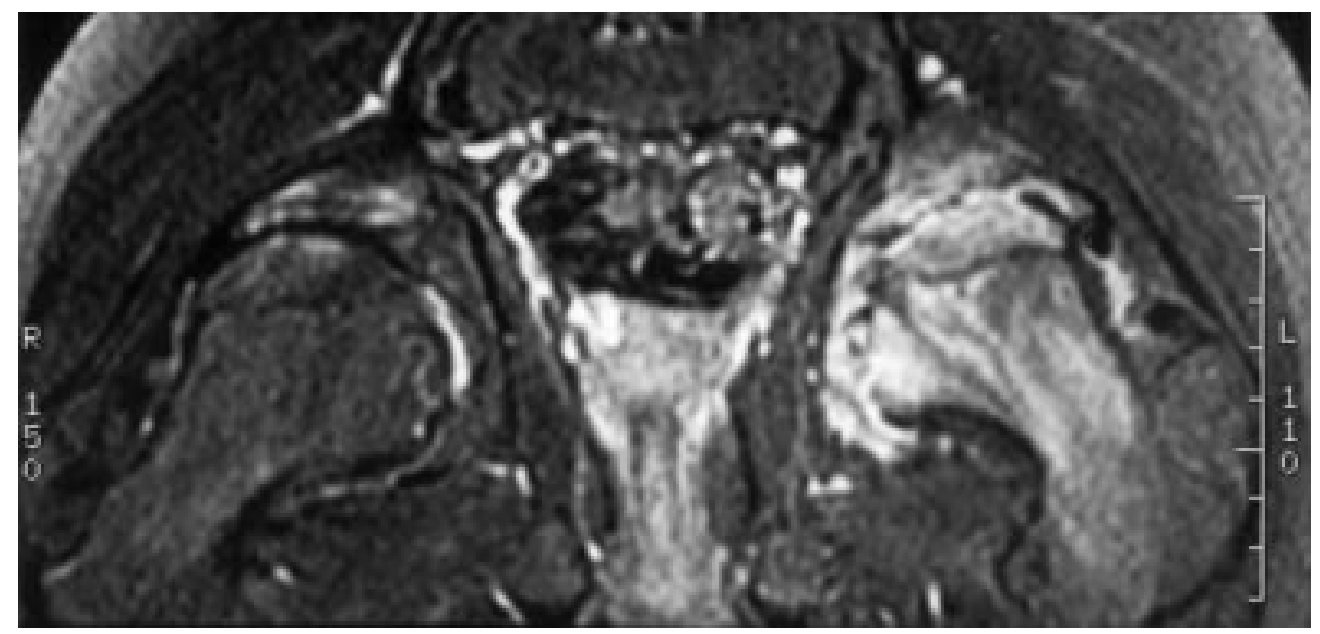

Figure 5 Patient No 3 aged 11 years. Gadolinium enhanced magnetic resonance imaging (T1 fat suppressed) of the hips - coronal view: synovial enhancement (white areas); irregularity of femoral heads, more so on left; effusion (black pockets around hips); hyperaemia in the left femoral head and neck. 

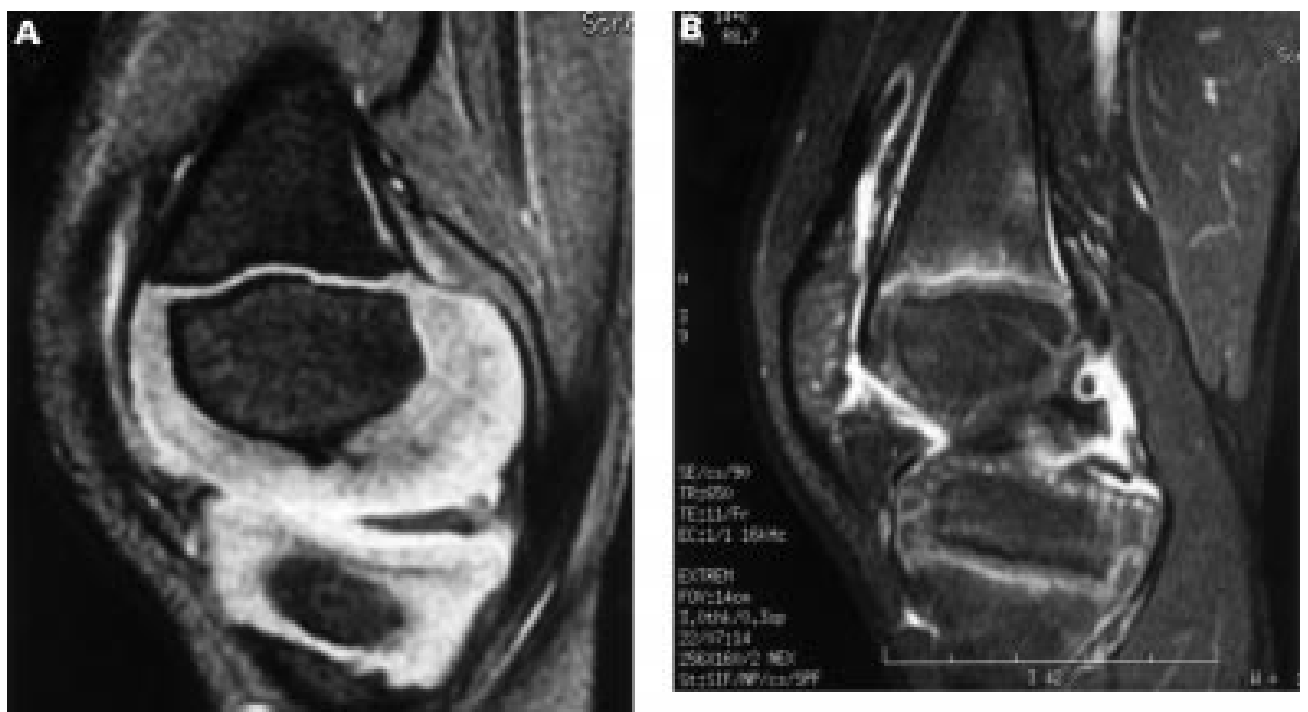

Figure 6 Patient No 4 aged 7 years. (A) Magnetic resonance imaging (gradient echo sequence) of the left knee-sagittal image: normal thickness and contour of the epiphysial and articular cartilage (white areas) of femoral condyles and proximal tibia. (B) Patient No 4 aged 7 years. Gadolinium enhanced magnetic resonance imaging (T1 fat suppressed) of left knee-sagittal image: synovial enhancement (white areas); small effusion best seen in suprapatellar pouch.

hip arthroplasty and is now awaiting arthroplasty for her right hip as well.

CASE 4

Patient No 4 is an $81 / 2$ year old boy who was diagnosed at 7 months of age with spondylometaphysial dysplasia based on radiological findings. At age 7 years he presented with a 6 to 12 month history of left knee pain and morning stiffness. On examination, his height was below the 5 th centile and weight on the 10 th centile. He had mild soft tissue swelling of the left knee and a $2 \mathrm{~cm}$ atrophy of his left quadriceps muscle. He also had a dorsal kyphosis and mild scoliosis.

On radiographic examination, he had severe platyspondyly with irregularity of the vertebral bodies, thoracic kyphosis, square-shaped horizontal pelvis with dysplasia of the acetabular roof, and shortening of the tubular bones with expanded metaphyses. On plain films, he had osteopenia but the joint space of the left knee was normal. MRI showed a small effusion of the left knee. The articular cartilage was normal on the gradient echo sequences (fig $6 \mathrm{~A})$. After gadolinium, there was synovial enhancement (fig 6B).

His ESR was as high as $30 \mathrm{~mm} / 1 \mathrm{st}$ h. He was $\mathrm{RF}$ negative, and ANA was positive at a titre of $1 / 40$. His symptoms improved significantly while he was taking NSAIDs. These symptoms returned when the drugs were stopped.

One year later he presented with severe left knee pain, different from the initial complaint. No effusion or soft tissue swelling was seen. An examination suggested a mechanical problem. Arthroscopy was done. It showed marked hypertrophy of the synovium, and degenerative changes of the femoral condyles and tibial plateau. Synovial biopsy showed minimum superficial chronic inflammation.

\section{Discussion}

This report describes the coexistence of inflammatory and degenerative arthritis in four patients with osteochondrodysplasias on both clinical and radiological grounds. To our knowledge, this is the first report to illustrate this coexistence. Although previous studies reported changes consistent with degenerative arthritis, ${ }^{1-16}$ none has highlighted radiographic changes of an inflammatory arthritis.

In our study these radiographic changes consisted of osteopenia, joint space narrowing in weightbearing and non-weightbearing joints, significant soft tissue swelling, bone hypertrophy secondary to chronic inflammation and hyperaemia, and erosions, especially those seen in the wrist in patient 2 . In addition, patient 1 had whiskering, which is a feature seen in spondyloarthropathies. Patient 3 had rapidly progressive joint space narrowing over 10 months. This progression was too rapid to be explained solely by a degenerative process.

MRI in children with JRA has been shown to provide an objective method of evaluating both synovial hypertrophy and the status of the articular cartilage. ${ }^{17}{ }^{18}$ Gadolinium enhanced MRI is sensitive in documenting active synovitis in children with JRA, especially in situations where the presence or absence of active synovitis could not be confirmed by the usual clinical and laboratory criteria. ${ }^{19}$ MRI was therefore done in these patients to verify the presence of active synovitis in specific joints. In all four patients MRI added additional information and clearly showed synovial hypertrophy and effusive disease. In patient 3 it also showed an extensive enhancing erosion of the left femoral head, which explains more clearly the rapidity of her course. Patient 4 had synovial enhancement and effusion, as well as normal articular cartilage seen on the gradient echo sequence of the MRI. These findings therefore cannot be explained solely by a degenerative arthritis as the articular cartilage was intact. In patients with osteochondrodysplasias who do not have an inflammatory arthritis associated with their disease, no effusion or synovial enhancement with gadolinium is seen. 
It is well known that these patients undergo premature osteoarthritis or degenerative arthritis. ${ }^{136911-1315}$ However, the clinical and radiological findings noted in our four patients cannot be explained solely by a degenerativetype of arthritis. Osteoarthritis or degenerative arthritis occurs later in life, usually during the second to fourth decade of life in this group, ${ }^{6}$ and commonly affects large weightbearing joints. In this report all four patients had onset of arthritis during the first decade of life. Additionally, patients 1 and 2 had a polyarticular course with clinical and radiological findings in both weightbearing and non-weightbearing joints. A degenerative arthritis cannot be the sole explanation when such findings occur before the first decade of life, affect nonweightbearing joints, and have a polyarticular course. Furthermore, the radiological evidence presented in this paper suggests that a degenerative and inflammatory arthritis coexist, which explains the above clinical findings. In addition, Patrone and Kredich have also suggested that these two entities occur in patients with multiple epiphysial dysplasia, another type of osteochondrodysplasia. ${ }^{6}$

Although the clinical and radiological findings depicted by these four cases support the presence of an inflammatory condition, we were unable to support this with laboratory evidence. One patient had an intermittent, mildly raised ESR, while in another the ESR was clearly raised (patient 4). The patients who underwent a synovial biopsy showed minimum chronic inflammation, and one of these patients also had a non-inflammatory synovial fluid. However, this does not preclude the presence of an ongoing inflammatory process as even patients with an inflammatory-type arthritis, such as pauciarticular juvenile arthritis, often do not have a raised ESR.

Most patients described in the literature are treated with NSAIDs ${ }^{2610}$ and physiotherapy. ${ }^{2610}$ All four patients received some benefit from this standard treatment. The first two patients in this case series were also treated with methotrexate. Their symptoms then decreased and, for the first time, some clinical improvement was seen, suggesting the presence of an inflammatory process.

By labelling these patients with a degenerative arthritis alone, their disease may not necessarily be treated as aggressively as one would treat a patient with an inflammatory arthritis. In many of these patients their disease certainly does behave aggressively enough to warrant consideration of second line agents.
In conclusion, the clinical and imaging findings of the four patients in this case series support the theory that both a degenerative and inflammatory arthritis can occur and coexist in patients with osteochondrodysplasias. It is important to recognise the inflammatory arthritis, which can be more difficult to appreciate in these patients, so that appropriate management can be started.

Dr R Scuccimarri is a clinical fellow of the Arthritis Society (Canada). Dr CM Duffy is a senior research scholar of the Arthritis Society (Canada).

1 Stanescu V, Stanescu R, Maroteaux P. Articular degeneration as a sequela of osteochondrodysplasias. Clin Rheum Dis 1985;11:239-70

2 Lewkonia RM, Bech-Hansen NT. Spondyloepiphyseal dysplasia tarda simulating juvenile arthritis: clinical and molecular genetic observations. Clin Exp Rheumatol 1992; 10:411-14.

3 Stanescu R, Stanescu V, Bordat C, Maroteaux P. Pathologic features of the femoral heads in a patient aged $14^{1 / 2}$ years with spondyloepiphyseal dysplasia with osteoarthritis. J wheumatol 1987;14:1061-7.

4 Al-Awadi SA, Farag TI, Naguib K, El-Khalifa MY, Cuschieri A, Hosny G, et al. Spondyloepiphyseal dysplasia tarda with progressive arthropathy. J Med Genet 1984;21:193-6.

5 Teebi AS, Al Awadi SA. Spondyloepiphyseal dysplasia tarda with progressive arthropathy: a rare disorder frequently diagnosed among Arabs. J Med Genet 1986;23:189-91.

6 Patrone NA, Kredich DW. Arthritis in children with multiple epiphyseal dysplasia. J Rheumatol 1985;12:145-9.

7 El-Shanti HE, Omari HZ, Qubain HI. Progressive pseudorheumatoid dysplasia: report of a family and review. J Med Genet 1997;34:559-63.

8 Wynne-Davies R, Hall C, Ansell BM. Spondylo-epiphysial dysplasia tarda with progressive arthropathy. J Bone Joint Surg Br 1982;64:442-5.

9 Spranger J, Albert C, Schilling F, Bartsocas C. Progressive pseudorheumatoid arthropathy of childhood (PPAC): a hereditary disorder simulating juvenile rheumatoid arthritis. Am J Med Genet 1983;14:399-401.

10 Spranger J, Albert C, Schilling F, Bartsocas C, Stöss H. Progressive pseudorheumatoid arthritis of childhood (PPAC): a hereditary disorder simulating rheumatoid arthritis. Eur J Pediatr 1983;140:34-40.

11 Lewkonia RM. The arthropathy of hereditary arthroophthalmopathy (Stickler syndrome). J Rheumatol 1992; 19:1271-5.

12 Spranger J. The epiphyseal dysplasias. Clin Orthop 1976;114:46-60.

13 Rai A, Wordsworth P, Coppock JS, Zaphiropoulos GC, Struthers GR. Hereditary arthro-ophthalmopathy (Stickler syndrome): a diagnosis to consider in familial premature osteoarthritis. Br J Rheumatol 1994;33:1175-80.

14 Kozlowski K, Kennedy J, Lewis IC. Radiographic features of progressive pseudorheumatoid arthritis. Australas Raof progressive pseudo
diol 1986;30:244-50.

15 Treble NJ, Jensen FO, Bankier A, Rogers JG, Cole WG. Development of the hip in multiple epiphseal dysplasia. J Bone Joint Surg Br 1990;72:1061-4.

16 Leguis E, Mulier M, Van Damme B, Fryns JP. Progressive pseudorheumatoid arthritis of childhood (PPAC) and normal adult height. Clin Genet 1993;44:152-5.

17 Yulish BS, Lieberman JM, Newman AJ, Bryan PJ, Mulopulos GP, Modic MT. Juvenile rheumatoid arthritis: assessment with MR imaging. Radiology 1987;165:149-52.

18 Hervé-Somma CMP, Sebag GH, Prieur AM, Bonnerot V, Lallemand DP. Juvenile rheumatoid arthritis of the knee: MR evaluation with Gd-DOTA. Radiology 1992;182: 93-8

19 Scuccimarri R, Watanabe Duffy KN, Azouz EM, Duffy $\mathrm{CM}$. Gadolinium enhanced magnetic resonance imaging in juvenile arthritis [abstract]. J Rheumatol 1999;26:1629. 